\title{
Methicillin-Resistant Staphylococcus Aureus Bacteremia: Epidemiology, Clinical Characteristics, Risk Factors, and Outcomes in a Tertiary Care Center in Riyadh, Saudi Arabia
}

Sarah A. Alhunaif ${ }^{1}$, Sarah Almansour ${ }^{2}$, Raghad Almutairi ${ }^{1}$, Sara Alshammari ${ }^{1}$, Lamia Alkhonain ${ }^{1}$, Bassam Alalwan ${ }^{3}$, Sameera Aljohani ${ }^{3}$, Abdulrahman Alsaedy ${ }^{2}$, Mohammad Bosaeed ${ }^{2}$, Adel Alothman ${ }^{4}$

1. College of Medicine, King Saud bin Abdulaziz University for Health Sciences, Riyadh, SAU 2. Division of Infectious Diseases, Department of Internal Medicine, King Abdulaziz Medical City - National Guards Health Affairs, Riyadh, SAU 3. Department of Pathology \& Laboratory Medicine, King Abdulaziz Medical City - National Guards Health Affairs, Riyadh, SAU 4. Department of Infectious Diseases, King Abdullah International Medical Research Center, Riyadh, SAU

Corresponding author: Sarah A. Alhunaif, alhunaif.sarah96@gmail.com

\section{Abstract \\ Background}

Methicillin-resistant Staphylococcus aureus (MRSA) has become increasingly common in hospitals worldwide. In an era of pandemics, it is important to understand all types of infectious diseases in order to control its outcome and reduce mortality rates related to it. This study aims to identify the epidemiology of MRSA bloodstream infection, risk factors, and mortality in King Abdulaziz Medical City in Riyadh.

\section{Methods}

This is a retrospective chart review study from January 2013 to June 2017. All patients with positive blood culture for MRSA at King Abdulaziz Medical City (KAMC), which is a tertiary care center in Riyadh, Saudi Arabia, were included. Data were extracted from electronic and paper medical records for patients who had a positive blood culture for MRSA. Data collected included demographics, microbiological details, risk factors, and 30-day mortality.

\section{Results}

From 2013 through June 2017, 633 Staphylococcus aureus bacteremia were reported in KAMC, of which 184 (29.1\%) were MRSA with a mean age of $60 \pm 19$ years. Almost all of our isolates were susceptible to vancomycin, linezolid, and tigecycline. The most common infectious syndrome these patients presented with was an endovascular infection which accounts for $30.4 \%$ while $19.9 \%$ presented as a case of pneumonia. The mortality within 30 days of collection of the positive blood culture was $20.65 \%$. Male gender $(\mathrm{OR}=2.33$; $95 \% \mathrm{CI}=1.34-4.05$; P-value $=0.003)$ and patients with history of recent hospital $(\mathrm{OR}=2.34 ; 95 \% \mathrm{CI}=1.27-$

Review began 04/19/2021 Review ended 05/05/2021 Published 05/10/2021

\section{() Copyright 2021}

Alhunaif et al. This is an open access article distributed under the terms of the Creative Commons Attribution License CC-BY 4.0., which permits unrestricted use, distribution, and reproduction in any medium, provided the original author and source are credited. 4.34; $\mathrm{P}$-value $=0.007)$ or ICU $(\mathrm{OR}=1.66 ; 95 \% \mathrm{CI}=1.09-2.52 ; \mathrm{P}$-value $=0.018)$ admissions were more likely to acquire MRSA.

\section{Conclusions}

The incidence of MRSA bacteremia at King Abdulaziz Medical City is high and increasing in conjunction with incidence rate posing a significantly high rate of mortality. Recent history of hospital admission, ICU admission and males were found to be significantly associated with higher rates of MRSA infection. Some modifiable risk factors that could be used to facilitate the reduction of MRSA acquisition rates is to avoid unnecessary hospital and ICU admissions.

Categories: Internal Medicine, Infectious Disease, Epidemiology/Public Health

Keywords: methicillin resistant staphylococcus aureus (mrsa), incidence, risk factors, mortality, bacteremia, methicillin resistant staphylococcus aureus bacteremia

\section{Introduction}

Methicillin-resistant Staphylococcus aureus (MRSA) is a significant global healthcare problem associated with remarkable morbidity and mortality among infected patients. MRSA accounts for most of the global Staphylococcus aureus (S. aureus) bacteremia cases. In comparison with methicillin-sensitive $S$. aureus (MSSA), MRSA infections have a higher rate of undesirable clinical outcomes including prolonged hospital stay, metastatic infections such as infective endocarditis, septic arthritis, osteomyelitis, and death [1]. An Australian study was conducted for over one year concluded that 450 of 1994 cases (24\%) of S. aureus bacteremia were due to MRSA. Most importantly, this study showed that 30-day mortality was $30 \%$ due to MRSA compared with $17.7 \%$ for MSSA [2]. 
MRSA bacteremia has majorly increased in recent years due to the increased prevalence of invasive procedures, increased numbers of immunosuppressed patients, as well as the increased resistance to antibiotics [3]. Many studies have found that the prevalence of MRSA is influenced by geographical location. It is now as high as up to $60 \%$ in certain centers in the United States [4], and more than $70 \%$ in a report from Shanghai, [5] but great geographic variations exist worldwide. In European centers, these percentages varied from less than $2 \%$ in the Netherlands to $54.4 \%$ in Portugal [6]. In the US hospitals, $60 \%$ of $S$. aureus isolates are found in the intensive care unit (ICU) [7]. MRSA in non-ICU patients annual rate in the USA has increased during the period 1998-2005 to 59.2\% [4]. Several studies showed several factors associated with $S$. aureus bacteremia including previous MRSA infection or colonization, skin ulcers or cellulitis at hospital admission, the presence of a central venous catheter, urinary catheter insertion, skin and soft tissue infection, intravenous drug abuse, the presence of the immunocompromising condition, use of corticosteroid and liver disease [8].

In Saudi Arabia, a meta-analysis was done for epidemiological data from 26 studies from across Saudi Arabia during 2002-2012. It showed that MRSA prevalence rate increased from $2 \%$ in 1988 reaching up to $38 \%$ in 2012 [9]. Meanwhile, an older study was conducted in 1998 at King Abdulaziz University Hospital in Jeddah, Saudi Arabia reported that $74.8 \%$ of MRSA isolates were obtained from hospitals. Surgical wounds (31.1\%), the chest (27\%), and endovascular catheters (20.3\%) are frequently the common sites of infection [10].

This study aims to determine the epidemiology of MRSA bacteremia from 2013 following up to 2017, by describing the demography and clinical characteristics of patients, identifying modifiable risk factors, and estimating the 30-day mortality.

\section{Materials And Methods Design and data collection}

This is a retrospective cohort study consisting of 184 blood samples positive for MRSA in King Abdulaziz Medical City in Riyadh, which is a tertiary care center with a bed capacity of approximately 1,200 beds serving a steady population of the national guard, military personnel, and civilians and their dependents. All adult patients with positive blood culture for MRSA who were admitted were included in the study. We collected all samples between January 2013 until June 2017. Patient characteristics included gender, age, patient's comorbidities, recent hospital admission, Intensive care unit admissions, length of hospitalization, department of admission, an infectious syndrome that the patient was diagnosed with and the presence of a prosthetic device or central venous catheter, microbiological data and mortality. All data collected was archived by the institutional review board with access provided on request. An episode of MRSA bacteremia was defined as any blood culture positive with MRSA. Episodes occurring within two weeks of a previous one, or without resolution of signs and symptoms of infection after a previous episode, were considered as single episodes. An intravascular device (IVD) was thought the likely focus of infection if there was evidence of inflammation at the catheter insertion site and/or catheter tip culture was positive for MRSA by a semiquantitative method. Endocarditis was defined according to the modified Duke's criteria. Pneumonia was defined as the source of bacteremia in the presence of the purulent sputum from which MRSA was isolated and new lung field opacity on radiographic examination.

\section{Statistical analysis}

All data were analyzed using R software version 4.0.2 (R Foundation for Statistical Computing, Vienna, Austria) [11] using the packages (Rcmdr) [12] and (glm2) [13]. Categorical variables were represented as frequencies, and percentages with Chi-square test (or Fisher's exact test, as appropriate) was used for testing the gender differences. For continuous variables, the representation was as means and standard deviations, using skewness and Kurtosis tests to evaluate the normal distribution of the variables. Based on normality status, independent-samples t-test or Mann-Whitney U-test were used to compare females to males. Moreover, we used univariate logistic regression to identify any possible modifiable risk factors for MRSA. Logistic regression results were expressed as odds ratios (ORs) and 95\% confidence interval (95\% CI). A Pvalue of $\leqslant 0.05$ was considered statistically significant in all analyses.

\section{Results}

During the study period, a total number of 633 S. aureus isolates were isolated from blood; of which 184 (29.1\%) were MRSA. Among MRSA patients, the number of male patients was 124 (67.4\%) whereas female patients 60 (32.6\%), with a mean age of $60 \pm 19$ years. Regarding co-morbidities, 127 (69.0\%) of the patients were hypertensive, 120 (65.2\%) were diabetics, 57 (31.0\%) were suffering from chronic kidney disease, 26 (14.1\%) were diagnosed with cancer, 8 (4.3\%) were on long-term corticosteroid usage and $4(2.2 \%)$ had an autoimmune disease. It should be noted that $45.7 \%$ of the patients have a history of recent hospital admissions during the last 90 days and 66 (35.9\%) patients had documented a previous history of MRSA colonization and/or infection. (Table 1). 


\begin{tabular}{|c|c|c|c|c|c|c|c|c|}
\hline \multirow{2}{*}{ Variables } & & \multicolumn{4}{|c|}{$\begin{array}{l}\text { Previous history of colonization/infection with } \\
\text { MRSA }\end{array}$} & \multicolumn{2}{|c|}{ Total } & \multirow{2}{*}{$\begin{array}{l}\text { P- } \\
\text { value }\end{array}$} \\
\hline & & $\mathrm{n}$ & $\%$ & $\mathrm{n}$ & $\%$ & $\mathrm{n}$ & $\%$ & \\
\hline \multicolumn{2}{|l|}{ Age; mean \pm SD } & \multicolumn{2}{|c|}{$57 \pm 18$} & \multicolumn{2}{|c|}{$61 \pm 19$} & \multicolumn{2}{|c|}{$60 \pm 19$} & 0.135 \\
\hline \multirow{2}{*}{ Gender } & Male & 48 & 72.7 & 76 & 64.4 & 124 & 67.4 & \multirow{2}{*}{0.248} \\
\hline & Female & 18 & 27.3 & 42 & 35.6 & 60 & 32.6 & \\
\hline \multirow{5}{*}{ Year of admission } & 2013 & 11 & 16.7 & 24 & 20.3 & 35 & 19.0 & \multirow{5}{*}{0.507} \\
\hline & 2014 & 12 & 18.2 & 19 & 16.1 & 31 & 16.8 & \\
\hline & 2015 & 4 & 6.1 & 16 & 13.6 & 20 & 10.9 & \\
\hline & 2016 & 26 & 39.4 & 38 & 32.2 & 64 & 34.8 & \\
\hline & 2017 & 13 & 19.7 & 21 & 17.8 & 34 & 18.5 & \\
\hline \multirow{2}{*}{ Diabetes mellitus } & Yes & 43 & 65.2 & 77 & 65.3 & 120 & 65.2 & \multirow{2}{*}{0.989} \\
\hline & No & 23 & 34.8 & 41 & 34.7 & 64 & 34.8 & \\
\hline \multirow{2}{*}{ Hypertension } & Yes & 49 & 74.2 & 78 & 66.1 & 127 & 69.0 & \multirow{2}{*}{0.252} \\
\hline & No & 17 & 25.8 & 40 & 33.9 & 57 & 31.0 & \\
\hline \multirow{2}{*}{ Chronic kidney disease } & Yes & 21 & 31.8 & 36 & 30.5 & 57 & 31.0 & \multirow{2}{*}{0.854} \\
\hline & No & 45 & 68.2 & 82 & 69.5 & 127 & 69.0 & \\
\hline \multirow{2}{*}{ On long-term corticosteroids } & Yes & 4 & 6.1 & 4 & 3.4 & 8 & 4.3 & \multirow{2}{*}{0.460} \\
\hline & No & 62 & 93.9 & 114 & 96.6 & 176 & 95.7 & \\
\hline \multirow{2}{*}{ Autoimmune disease } & Yes & 1 & 1.5 & 3 & 2.5 & 4 & 2.2 & \multirow{2}{*}{1.00} \\
\hline & No & 65 & 98.5 & 115 & 97.5 & 180 & 97.8 & \\
\hline \multirow{2}{*}{ Cancer } & Yes & 10 & 15.2 & 16 & 13.6 & 26 & 14.1 & \multirow{2}{*}{0.766} \\
\hline & No & 56 & 84.8 & 102 & 86.4 & 158 & 85.9 & \\
\hline \multirow{2}{*}{$\begin{array}{l}\text { History of recent hospital admission (last } 90 \\
\text { days) }\end{array}$} & Yes & 39 & 59.1 & 45 & 38.1 & 84 & 45.7 & \multirow{2}{*}{$0.006^{*}$} \\
\hline & No & 27 & 40.9 & 73 & 61.9 & 100 & 54.3 & \\
\hline
\end{tabular}

TABLE 1: Summary of baseline characteristics of the included patients $(N=184)$.

MRSA: methicillin-resistant Staphylococcus aureus, N: numbers; SD: standard deviation.

*Statistically significant.

The type of infectious syndrome varied among the population study, 56 (30.4\%) were presented as cases of endovascular infection such as septic thrombophlebitis, endocarditis, central venous catheter infections. Pneumonia was reported in 36 (19.6\%) of the patients, 35 (19.0\%) of the patients were presented with skin and soft tissue infections, 24 (13.0\%) had bone and/or joint infection, 19 (10.3\%) with urinary tract infection, and in 12 (6.5\%) patients there was no clear source of infection identified. At the time of diagnosis, 88 (47.8\%) were having a central venous catheter, 11 (6.0\%) had implanted a medical prosthetic device, and 52 (28.3\%) had surgery during the same admission (Table 2). 


\section{Cureus}

\begin{tabular}{|c|c|c|c|}
\hline Variable & & $\mathbf{N}$ & $\%$ \\
\hline \multirow{4}{*}{ Specialty } & Medicine & 132 & 71.7 \\
\hline & Surgery & 35 & 19.0 \\
\hline & Obstetrics & 1 & 0.5 \\
\hline & Oncology & 16 & 8.7 \\
\hline \multirow{7}{*}{ Infectious syndromes } & Pneumonia & 36 & 19.6 \\
\hline & Endovascular & 56 & 30.4 \\
\hline & Bone/joint & 24 & 13.0 \\
\hline & Skin and soft tissue & 35 & 19.0 \\
\hline & UTI & 19 & 10.3 \\
\hline & CNS & 2 & 1.1 \\
\hline & Unknown source & 12 & 6.5 \\
\hline \multirow{2}{*}{ Central venous catheter } & Yes & 88 & 47.8 \\
\hline & No & 96 & 52.2 \\
\hline \multirow{2}{*}{ Implanted medical prosthetic device* } & Yes & 11 & 6.0 \\
\hline & No & 173 & 94.0 \\
\hline \multirow{2}{*}{ Had surgery during the same admission } & Yes & 52 & 28.3 \\
\hline & No & 132 & 71.7 \\
\hline Length of hospitalization (days); mean \pm SD & & \multicolumn{2}{|c|}{$55 \pm 112$} \\
\hline
\end{tabular}

TABLE 2: Summary of clinical characteristics of the patients $(N=184)$.

UTI: urinary tract infection; CNS: central nervous system; N: numbers; *including: cardiac device, prosthetic valve, knee prosthetic joint, vertebrae prosthesis; SD: standard deviation.

A total of 91 (49.46\%) of the patients were admitted to the intensive care unit at the time of diagnosis. The majority of the patients were admitted under medical specialty 132 (71.74\%), 36 (19.56\%) under surgical specialty, 16 (8.7\%) under hematology/oncology service and 1 patient admitted with obstetrics and gynecology (0.5\%). The 30-day mortality for the patients with MRSA bacteremia was (20.1\%) 35/174, 10 patients were lost to follow-up (Figure 1). 


\section{Cureus}

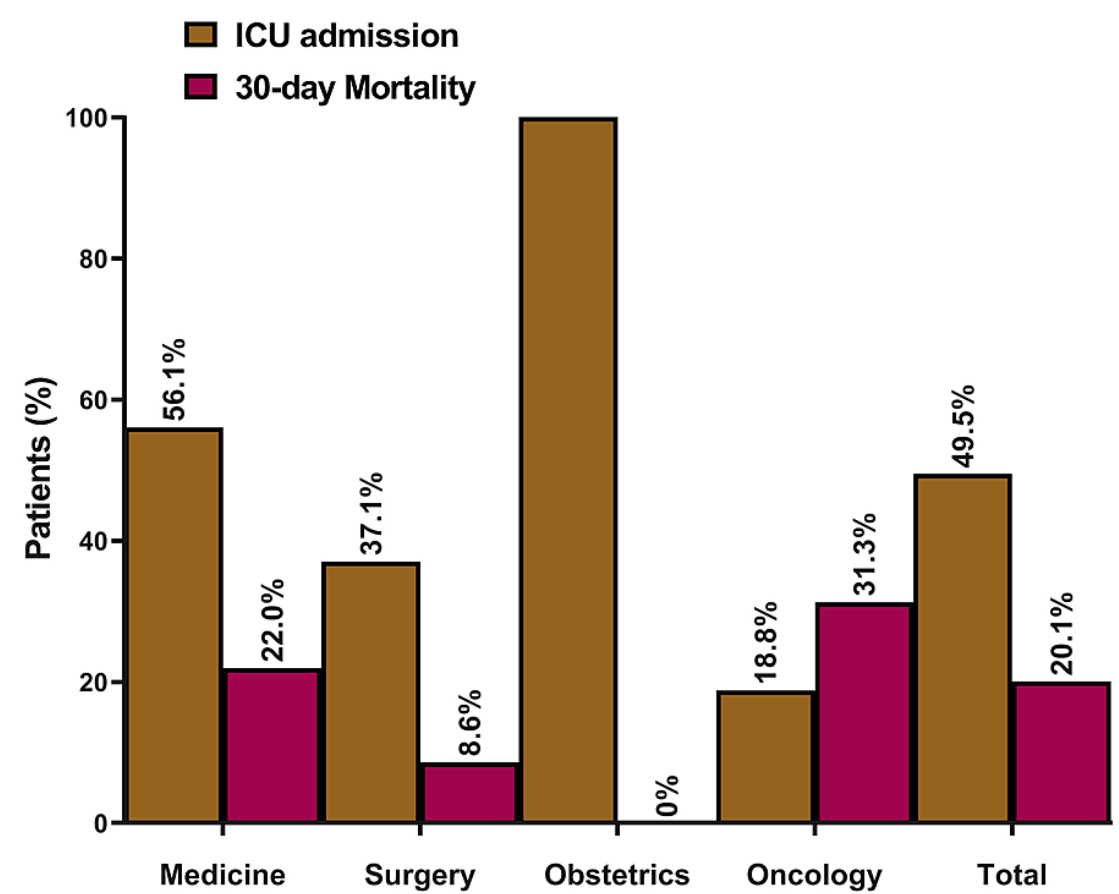

FIGURE 1: MRSA patients' outcomes (ICU admission and 30-day mortality), stratified by admitting specialties $(\mathrm{N}=184)$.

MRSA: methicillin-resistant Staphylococcus aureus; ICU: intensive care unit; N: numbers.

Almost all MRSA isolates in our study were susceptible in vitro to vancomycin, linezolid, and tigecycline. Susceptibility to other antibiotics was variable with $95.1 \%$ of the isolates susceptible to nitrofurantoin, $75.5 \%$ to moxifloxacin, $73.9 \%$ to gentamicin, $58.2 \%$ to clindamycin, and $55.4 \%$ to erythromycin (Figure 2 ).

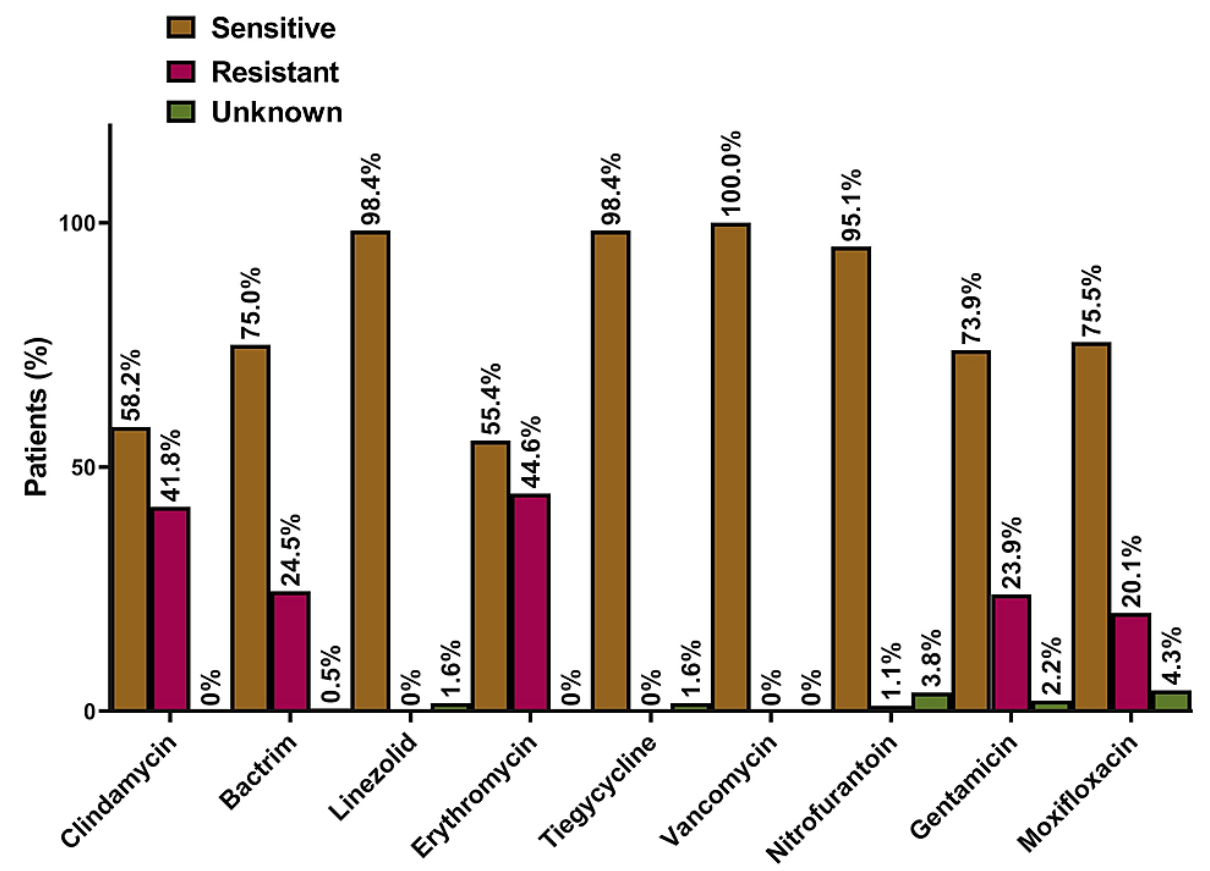

FIGURE 2: Antimicrobial susceptibility of MRSA isolates ( $N=184)$.

MRSA: methicillin-resistant Staphylococcus aureus; N: numbers. 
Table 3 presents results from the univariate logistic analysis of different factors among MRSA and nonacquisition groups. On one hand, male gender $(\mathrm{OR}=2.33 ; 95 \% \mathrm{CI}=1.34-4.05 ; \mathrm{P}$-value $=0.003)$ and patients with history of recent hospital $(\mathrm{OR}=2.34 ; 95 \% \mathrm{CI}=1.27-4.34 ; \mathrm{P}$-value $=0.007)$ or $\mathrm{ICU}(\mathrm{OR}=1.66 ; 95 \% \mathrm{CI}=$ 1.09-2.52; P-value $=0.018$ ) admissions were more likely to acquire MRSA. On the other hand, patients who were on long-term corticosteroids $(\mathrm{OR}=0.54 ; 95 \% \mathrm{CI}=0.40-0.74 ; \mathrm{P}$-value $<0.001)$, those with an autoimmune disease $(\mathrm{OR}=0.57 ; 95 \% \mathrm{CI}=0.42-0.77$; $\mathrm{P}$-value $<0.001)$, and those who had surgery during the same admission $(\mathrm{OR}=0.31 ; 95 \% \mathrm{CI}=0.16-0.60 ; \mathrm{P}$-value $<0.001)$ showed lower frequency of MRSA infections. In contrast, age, co-morbidities, cancer, admitting specialty, having central venous catheter, or implanted medical prosthesis did not show any significant association with MRSA rates.

\begin{tabular}{|c|c|c|c|c|c|}
\hline \multirow{2}{*}{ Predictor } & \multirow{2}{*}{ Estimate } & \multirow{2}{*}{ P-value } & \multirow{2}{*}{ Odds ratio } & \multicolumn{2}{|c|}{$95 \%$ confidence interva } \\
\hline & & & & Lower & Upper \\
\hline Gender (male versus female) & 0.85 & $0.003^{*}$ & 2.33 & 1.34 & 4.05 \\
\hline Age & -0.01 & 0.257 & 0.99 & 0.98 & 1.01 \\
\hline Diabetes mellitus & 0 & 0.989 & 1 & 0.53 & 1.87 \\
\hline Hypertension & 0.39 & 0.253 & 1.48 & 0.76 & 2.89 \\
\hline Chronic kidney disease & 0.06 & 0.854 & 1.06 & 0.56 & 2.04 \\
\hline On long-term corticosteroids & -0.61 & & 0.54 & 0.40 & 0.74 \\
\hline Autoimmune disease & -0.57 & & 0.57 & 0.42 & 0.77 \\
\hline Cancer & 0.13 & 0.766 & 1.14 & 0.48 & 2.68 \\
\hline History of recent hospital admission (last 90 days) & 0.85 & $0.007^{*}$ & 2.34 & 1.27 & 4.34 \\
\hline Admitting specialty & 0.07 & 0.663 & 1.08 & 0.77 & 1.51 \\
\hline ICU admission & 0.51 & $0.018^{*}$ & 1.66 & 1.09 & 2.52 \\
\hline Central venous catheter & -0.23 & 0.454 & 0.79 & 0.43 & 1.45 \\
\hline Implanted medical prosthetic device & -0.02 & 0.972 & 0.98 & 0.28 & 3.47 \\
\hline Had surgery during the same admission & -1.17 & & 0.31 & 0.16 & 0.60 \\
\hline
\end{tabular}

TABLE 3: Univariate logistic regression of potential risk factors for methicillin-resistant Staphylococcus aureus.

SE: standard error; ICU: intensive care unit.

*Statistically significant.

\section{Discussion}

The current study is one of the few studies on MRSA epidemiology in a tertiary-care Saudi setting. We conducted a longstanding cohort of all adult patients with positive blood culture for MRSA who were admitted to King Abdulaziz Medical City between January 2013 until June 2017. The results of our study showed that a high MRSA incidence of $29.1 \%$, which is consistent with the reported incidence in literature, ranging from $7 \%$ to $60 \%$ [14].

Approximately one-third (30.4\%) of cases presented as an endovascular infection, which represented the most common site of infection among the bacteremia patients followed by pneumonia and skin and soft tissue infection (19.9\%) (19.3\%), respectively. The increased susceptibility of having an endovascular infection could be related to the poor general condition of patients in the present study, as most patients had comorbidities, other reason would be the presence of intravascular devices in most of these patients. These results were similar to what has been observed by other researchers. For instance, in the Veterans Administration Medical Centre, Buffalo, New York, the commonest foci of Staphylococcus aureus bacteremia (SAB) were intravascular catheter (33\%), postoperative wounds (11\%), skin infections (7\%), pulmonary infections (7\%) [15]. Moreover, sensitivities to a selection of anti-staphylococcal antibiotics were available from routine automated testing. All isolates were sensitive to vancomycin, linezolid, and tigecycline and variable susceptibility to other agents. Similar results were observed in other studies [16]. 
Although the mean age of patients presented with bacteremia was 60 years, MRSA affected all age groups, which was also evident with age being an insignificant predictor for MRSA risk. Many previous studies of MRSA patients and healthy controls came to the same conclusion that age by itself may not be a significant risk factor for MRSA [17]. Contradicting with our findings, some other studies concluded that advanced age, especially those who are older than 65 years, are at more risk to acquire MRSA [18-21]. This may be explained by the increased risk of hospital admission and hence the risk of MRSA acquisition among senior age groups, which is not a direct effect $[22,23]$. In the same context, our results showed an increased risk of acquiring MRSA among male patients, which has been also confirmed by previous studies [18,20]. In one of the biggest cohorts of MRSA acquisition in an acute tertiary-care hospital, they found male gender to be a significant predictor (P-value $<0.001$ ) of MRSA bacteremia, compared to females [18]. Since the inappropriate hygienic habits can increase the risk of horizontal transmission of infections, which is consistent with MRSA strains, this higher incidence among males could be a reflection of their lower hygienic standards [24].

History of recent hospital admission and ICU admission were both significant predictors of higher risk of MRSA acquisition. Many previous studies showed that hospitalization is a major risk factor for MRSA acquisition which acts in a dose-response manner, based on the length of hospital stay [17,18,25]. In Singapore, a large case-control study of 1200 patients showed that hospitalization had a direct effect on MRSA acquisition and the length of stay accounted for the most of effects caused by age (100\%), surgical operations (96\%), and immunosuppressive states (67\%) [25]. Akin to that, ICU-admitted patients were at higher risk of nosocomial MRSA infections, compared to those admitted at non-ICU departments $[20,26]$. This may be explained by the increased need for mechanical ventilation in ICU-admitted patients, which is a known risk factor for ventilator-related pneumonia and, in turn, MRSA acquisition [27]. Other identified risk factors for the high prevalence in the ICU setting include inappropriate numbers of well-trained nurses and bad hygiene measures [26]. In the same context, our results showed a reduction in MRSA acquisition rates among patients with auto-immune diseases and those who are on a long-term corticosteroid. Although corticosteroid was found to increase the risk of $\mathrm{S}$. aureus acquisition in general and MRSA in specific [28], many other studies, like the current one, did not show any significant increase in MRSA acquisition rates among corticosteroid users [17].

The mortality rate within 30 days after the MRSA bacteremia episode during the study period was (21.05\%). Notably, we found that 30-day mortality has increased over time from (19.3\%) in 2013 to (38.2\%) in 2017 despite advancement in antimicrobial treatment. Our results were similar to a study that was done in a university hospital, Barcelona, Spain, which showed that the mortality of patients with MRSA bacteremia remains high, close to 30\% [29]. Also, a large study carried out across England during 2004-2005 showed that 30-day mortality due to MRSA bacteremia was (38\%) [30].

The limitations of the present study include the retrospective study design and the sensitivity of MRSA detection which is mainly dependent on the sensitivity of the culture. Nevertheless, these possible biases would not be eliminated when adopting the case and control groups. As limited by study design, there is a possible residual confounding and accordingly, the causation could not be established. Regarding our study strength, it is one of the largest cohort studies to be conducted in Saudi Arabia on MRSA acquisition, with a long-standing period of five years and taking into count many variables. Using patients' structured medical records would ensure data accuracy/consistency and minimize measurement mistakes. All collected data were not liable to recall bias or protocol variation since none of them was based on patient reporting.

\section{Conclusions}

The prevalence of MRSA bacteremia at King Abdulaziz Medical City is high and increasing and carries a significant rate of mortality. Recent history of hospital admission, ICU admission and males were found to be significantly associated with higher rates of MRSA infection. Some modifiable risk factors that could be used to facilitate the reduction of MRSA acquisition rates is to avoid unnecessary hospital and ICU admissions. Lastly, more studies are needed to explore the role of unmodifiable risk factors such as male gender in MRSA infection.

\section{Additional Information \\ Disclosures}

Human subjects: Consent was obtained or waived by all participants in this study. King Abdulaziz Medical City IRB issued approval NA. Patients' confidentiality was insured and no identifying information will be published and the study was approved by the Institutional Review Board (IRB) of King Abdulaziz Medical City. Animal subjects: All authors have confirmed that this study did not involve animal subjects or tissue. Conflicts of interest: In compliance with the ICMJE uniform disclosure form, all authors declare the following: Payment/services info: All authors have declared that no financial support was received from any organization for the submitted work. Financial relationships: All authors have declared that they have no financial relationships at present or within the previous three years with any organizations that might have an interest in the submitted work. Other relationships: All authors have declared that there are no other relationships or activities that could appear to have influenced the submitted work. 


\section{References}

1. Hassoun A, Linden PK, Friedman B: Incidence, prevalence, and management of MRSA bacteremia across patient populations-a review of recent developments in MRSA management and treatment. Crit Care. 2017, 21:211. 10.1186/s13054-017-1801-3

2. Davis JS, Sud A, O'Sullivan MVN, et al.: Combination of vancomycin and $\beta$-lactam therapy for methicillinresistant staphylococcus aureus bacteremia: a pilot multicenter randomized controlled trial. Clin Infect Dis. 2016, 62:173-80. 10.1093/cid/civ808

3. Naber CK: Staphylococcus aureus bacteremia: epidemiology, pathophysiology, and management strategies . Clin Infect Dis. 2009, 48 Suppl 4:S231-7. 10.1086/598189

4. Styers D, Sheehan DJ, Hogan P, Sahm DF: Laboratory-based surveillance of current antimicrobial resistance patterns and trends among Staphylococcus aureus: 2005 status in the United States. Ann Clin Microbiol Antimicrob. 2006, 5:2. 10.1186/1476-0711-5-2

5. Song Y, Du X, Li T, Zhu Y, Li M: Phenotypic and molecular characterization of Staphylococcus aureus recovered from different clinical specimens of inpatients at a teaching hospital in Shanghai between 2005 and 2010. J Med Microbiol. 2013, 62:274-82. 10.1099/jmm.0.050971-0

6. Diekema DJ, Pfaller MA, Schmitz FJ, Smayevsky J, Bell J, Jones RN, Beach M: Survey of infections due to Staphylococcus species: frequency of occurrence and antimicrobial susceptibility of isolates collected in the United States, Canada, Latin America, Europe, and the Western Pacific region for the SENTRY Antimicrobial Surveillance Program, 1997-1999. Clin Infect Dis. 2001, 32 Suppl 2:S114-32. 10.1086/320184

7. National Nosocomial Infections Surveillance (NNIS) System Report, data summary from January 1992 through June 2004, issued October 2004. Am J Infect Control. 2004, 32:470-85. 10.1016/S0196655304005425

8. Lewis T, Chaudhry R, Nightingale P, Lambert P, Das I: Methicillin-resistant Staphylococcus aureus bacteremia: epidemiology, outcome, and laboratory characteristics in a tertiary referral center in the UK. Int J Infect Dis. 2011, 15:e131-5. 10.1016/j.ijid.2010.09.013

9. Madani TA: Epidemiology and clinical features of methicillin-resistant Staphylococcus aureus in the University Hospital, Jeddah, Saudi Arabia. Can J Infect Dis. 2002, 13:245-50. 10.1155/2002/235213

10. Harbarth S, Rutschmann O, Sudre P, Pittet D: Impact of methicillin resistance on the outcome of patients with bacteremia caused by Staphylococcus aureus. Arch Intern Med. 1998, 158:182-9. 10.1001/archinte.158.2.182

11. Team RC: R: A Language and Environment for Statistical Computing . R Foundation for Statistical Computing, Vienna, Austria; 2017.

12. Fox J, Bouchet-Valat M, Andronic L, et al.: Package 'Rcmdr'. 2020,

13. Marschner I, Donoghoe MW: glm2: Fitting Generalized Linear Models. R package version 1.1. 2, Vienna, Austria; 2018.

14. Sabbagh P, Riahi SM, Gamble HR, Rostami A: The global and regional prevalence, burden, and risk factors for methicillin-resistant Staphylococcus aureus colonization in HIV-infected people: a systematic review and meta-analysis. Am J Infect Control. 2019, 47:323-3. 10.1016/j.ajic.2018.06.023

15. Mylotte JM, McDermott C, Spooner JA: Prospective study of 114 consecutive episodes of Staphylococcus aureus bacteremia. Rev Infect Dis. 1987, 9:891-907. 10.1093/clinids/9.5.891

16. El Amin NM, Faidah HS: Methicillin-resistant Staphylococcus aureus in the western region of Saudi Arabia: prevalence and antibiotic susceptibility pattern. Ann Saudi Med. 2012, 32:513-6. 10.5144/02564947.2012.513

17. Salangsang JA, Harrison LH, Brooks MM, Shutt KA, Saul MI, Muto CA: Patient-associated risk factors for acquisition of methicillin-resistant Staphylococcus aureus in a tertiary care hospital. Infect Control Hosp Epidemiol. 2010, 31:1139-47. 10.1086/656595

18. Loke HY, Kyaw WM, Chen MIC, Lim JW, Ang B, Chow A: Length of stay and odds of MRSA acquisition: a dose-response relationship?. Epidemiol Infect. 2019, 147:e223. 10.1017/S0950268819001110

19. Pobiega M, Chmielarczyk A, Pomorska-Wesołowska M, Ziolkowski G, Wojkowska-Mach J: Advanced age and long-term care facility stay as risk factors for methicillin-resistant Staphylococcus aureus infection. Antimicrob Resist Infect Control. 2015, 4:P191. 10.1186/2047-2994-4-S1-P191

20. Pomorska-Wesołowska M, Różańska A, Natkaniec J, et al.: Longevity and gender as the risk factors of methicillin-resistant Staphylococcus aureus infections in southern Poland. BMC Geriatr. 2017, 17:51. 10.1186/s12877-017-0442-3

21. van Velzen EV, Reilly JS, Kavanagh K, et al.: A retrospective cohort study into acquisition of MRSA and associated risk factors after implementation of universal screening in Scottish hospitals. Infect Control Hosp Epidemiol. 2011, 32:889-96. 10.1086/661280

22. Denkinger CM, Grant AD, Denkinger M, Gautam S, D'Agata EM: Increased multi-drug resistance among the elderly on admission to the hospital--a 12-year surveillance study. Arch Gerontol Geriatr. 2013, 56:227-30. 10.1016/j.archger.2012.05.006

23. Admi H, Shadmi E, Baruch H, Zisberg A: From research to reality: minimizing the effects of hospitalization on older adults. Rambam Maimonides Med J. 2015, 6:e0017. 10.5041/RMMJ.10201

24. Cianciara D, Miller M, Przewłocka T: Health behavior of Polish adult population. Przeglad Epidemiologiczny. 2002, 56:159-68.

25. Wong JG, Chen MI, Win MK, Ng PY, Chow A: Length of stay an important mediator of hospital-acquired methicillin-resistant Staphylococcus aureus. Epidemiol Infect. 2016, 144:1248-56. 10.1017/S0950268815002733

26. Dancer SJ, Coyne M, Speekenbrink A, Samavedam S, Kennedy J, Wallace PG: MRSA acquisition in an intensive care unit. Am J Infect Control. 2006, 34:10-17. 10.1016/j.ajic.2005.08.009

27. Rubinstein E, Kollef MH, Nathwani D: Pneumonia caused by methicillin-resistant Staphylococcus aureus . Clin Infect Dis. 2008, 46:S378-85. 10.1086/533594

28. Yamakawa K, Tasaki O, Fukuyama M, et al.: Assessment of risk factors related to healthcare-associated methicillin-resistant Staphylococcus aureus infection at patient admission to an intensive care unit in Japan. BMC Infect Dis. 2011, 11:303. 10.1186/1471-2334-11-303 


\section{Cureus}

29. Gasch O, Ayats J, Ángeles Dominguez M, et al.: Epidemiology of methicillin-resistant Staphylococcus aureus (MRSA) bloodstream infection: secular trends over 19 years at a university hospital. Medicine. 2011, 90:31927. 10.1097/MD.0b013e31822f0b54

30. Lamagni TL, Potz N, Powell D, Pebody R, Wilson J, Duckworth G: Mortality in patients with meticillinresistant Staphylococcus aureus bacteraemia, England 2004-2005. J Hosp Infect. 2011, 77:16-20. 10.1016/j.jhin.2010.07.015 